\title{
Recent Trials of Atrial Fibrillation Therapy
}

\author{
Baktash Morrad ${ }^{1}$ and Bulent Gorenek ${ }^{2}$
}

1. Staff Cardiologist; 2. Professor of Cardiology and Director, Department of Cardiology, Faculty of Medicine, Eskisehir Osmangazi University, Eskisehir, Turkey

\begin{abstract}
Atrial fibrillation (AF) is the most common sustained cardiac arrhythmia in clinical practice. It may cause significant symptoms and impair both functional status and quality of life. Without therapeutic intervention, affected patients are at increased risk of mortality and morbidity, so AF places a major burden on healthcare systems. Many trials have been published on AF therapy in recent years. In this editorial, we will briefly discuss recent trials of AF therapies.
\end{abstract}

\section{Keywords}

Atrial fibrillation, clinical trials

Disclosure: Baktash Morrad and Bulent Gorenek have no conflicts of interest to declare. No funding was received in the publication of this article. Open Access: This article is published under the Creative Commons Attribution Noncommercial License, which permits any non-commercial use, distribution, adaptation and reproduction provided the original author(s) and source are given appropriate credit.

Received: 16 April 2015 Published: 14 August 2015 Citation: European Journal of Arrhythmia \& Electrophysiology, 2015;1(1):17-8 DOI: 10.17925/EJAE.2015.01.01.17 Correspondence: Bulent Gorenek, Department of Cardiology, Faculty of Medicine, Eskisehir Osmangazi University, 26480 Eskisehir, Turkey. E: bulent@gorenek.com

Atrial fibrillation (AF) is the most common cardiac arrhythmia, which can have adverse consequences related to a reduction in cardiac output and to atrial and atrial appendage thrombus formation. ${ }^{1-4}$ The arrhythmia is associated with a fivefold risk of stroke and a threefold incidence of congestive heart failure and higher mortality.

The long-term outcomes, such as survival or rate of thromboembolism, are similar with either rhythm- or rate-control strategies in patients who have AF. In addition, anticoagulation is required with both in most patients. ${ }^{1,2}$ Thus the principal aim of therapy is to reduce symptoms by decreasing the frequency and duration of episodes. ${ }^{3,4}$ This article reviews recent studies of $\mathrm{AF}$ treatment.

In the TREAT-AF study, which evaluated mortality over 120,000 patients who were diagnosed with new non-valvular AF, $23.5 \%$ had received digoxin. Follow-up averaged around 3 years, and $23.5 \%$ of patients died during follow-up. Digoxin use was associated with mortality after multivariate adjustment (hazard ratio [HR] 1.26, $95 \%$ confidence interval [Cl] 1.23-1.29) and propensity matching (HR 1.21, $95 \% \mathrm{Cl} 1.17-1.25) .{ }^{5}$ In a post-hoc analysis of 14,171 patients in the ROCKET-AF trial database, digoxin was used at baseline in $37 \%$. After adjustment, digoxin use was associated with increased all-cause mortality (5.41 versus 4.30 events per 100 patient-years; HR 1.17, $95 \%$ Cl 1.04-1.32) over a median-duration follow-up of about 2 years. ${ }^{6}$

In a study comparing the effect of calcium channel blockers and betablockers on exercise capacity and reducing of B-type natriuretic peptide (NT-proBNP) levels on patient who had permanent AF, there were 60 patients (mean age $71 \pm 9$ years, 18 women) with permanent AF and normal left ventricular function in a randomised crossover investigatorblind study. Diltiazem 360 mg, verapamil 240 mg, metoprolol 100 mg and carvedilol $25 \mathrm{mg}$ were administered once daily (o.d.) for 3 weeks. At baseline and on the last day of each treatment period, the patients underwent a maximal cardiopulmonary exercise test, with blood samples obtained at rest and at peak exercise. The exercise capacity (peak $\mathrm{VO}_{2}$ ) was significantly lower during treatment using metoprolol and carvedilol than at baseline (no treatment) or with treatment using diltiazem and verapamil $(p<0.001$ for all). Compared with baseline, treatment with diltiazem and verapamil significantly reduced the NT-proBNP levels both at rest and at peak exercise, whereas treatment with metoprolol and carvedilol increased.?

Catheter ablation (CA) is an effective therapy for the treatment of symptomatic paroxysmal AF when medical therapy fails. However, its use in patients who have persistent AF remains controversial. The evidence in favour of ablation derives from non-randomised trials and is weak. The CA versus antiarrhythmic drug treatment of persistent AF (SARA) is the first multicentre, randomised, controlled trial. Patients who had persistent AF were randomly assigned to CA or antiarrhythmic drug therapy (ADT). The primary endpoint at 12-month follow-up was defined as any episode of AF or atrial flutter lasting $>24$ hours that occurred after a 3-month blanking period. Secondary endpoints were any atrial tachyarrhythmia lasting >30 seconds, hospitalisation and electrical cardioversion. In total, 146 patients were included (aged $55 \pm 9$ years, $77 \%$ male). The ADT group received class Ic (43.8\%) or class III drugs (56.3\%). In an intention-to-treat analysis, 69 of 98 patients (70.4 \%) in the CA group and 21 of 48 patients ( $43.7 \%$ ) in the ADT group were free of the primary endpoint $(\mathrm{p}=0.002)$, implying an absolute risk difference of $26.6 \%$ (95\% Cl 10.0-43.3) in favour of CA. The proportion of patients free of any recurrence ( $>30$ seconds) was higher in the CA group than in the ADT group (60.2 versus $29.2 \%$; $p<0.001$ ), and cardioversion was less frequent ( 34.7 versus $50 \%$, respectively; $\mathrm{p}=0.018$ ). There was periprocedural complication in six $(6.1 \%)$ in the CA group. ${ }^{8}$

Radiofrequency Ablation vs Antiarrhythmic Drugs as First-line Treatment for Paroxysmal Atrial Fibrillation (RAAFT-2) was a randomised clinical 
trial involving treatment of 127 patients with paroxysmal AF and showed that among patients with paroxysmal AF who had not previously had antiarrhythmic drug treatment, radiofrequency ablation compared with antiarrhythmic drugs resulted in a lower rate of recurrent atrial tachyarrhythmias at 2 years. However, recurrence was frequent in both groups. ${ }^{9}$

The prospective, multicentre, single-blinded study comparison of radiofrequency CA of drivers and circumferential pulmonary vein isolation in AF (RADAR-AF) randomised 232 patients who had paroxysmal AF $(n=115)$ to circumferential pulmonary vein isolation (CPVI) or high-frequency source ablation (HFSA) only (noninferiority design) and those with persistent $A F(n=117)$ to $C P V I$ or a combined ablation approach (CPVI + HFSA, superiority design). In paroxysmal AF, HFSA failed to achieve non-inferiority at 6 months after a single procedure but after redo procedures were non-inferior to CPVI at 12 months for freedom from AF and atrial flutter/atrial tachyarrhythmias. Serious adverse events were significantly reduced in the HFSA group versus CPVI patients ( $p=0.02$ ). In persistent $A F$, there were no significant differences between treatment groups for primary and secondary endpoints, but CPVI + HFSA trended towards more serious adverse events. ${ }^{10}$

According to studies, patients who undergo $\mathrm{CA}$ as a result of AF experience atrial tachycardias in the first few months, and it is also related to long-time recurrence of AF. In the AMIO-CAT study recurrence of arrhythmia subsequent to short-term oral amiodarone after CA for $A F$ randomised 212 patients who underwent $A F$ ablation. Patients were stratified according to type of AF (paroxysmal/persistent) and history of previous AF ablation and randomly assigned to 8 weeks of oral amiodarone therapy or to matched placebo subsequent to CA. Patients were followed for 6 months. Of 212 enrolled patients (50\% paroxysmal, $29 \%$ with history of previous ablation). Analyses were performed according to the intention-to-treat principle. Two hundred and six patients were available for analysis of the primary end-point, which was documented atrial tachyarrhythmia lasting $>30$ seconds subsequent to a blanking period of 3 months. This was observed in $42 / 107$ (39\%) in the amiodarone group versus $48 / 99(48 \%)$ in the placebo group ( $p=0.18$ ). Among the secondary endpoints, the amiodarone group showed a significantly lower rate of atrial tachyarrhythmia-related hospitalisations (rate ratio [RR] $0.43 ; 95 \% \mathrm{Cl} 0.23-0.77 ; \mathrm{p}=0.006$ ) and cardioversions (CA $0.36 ; 95 \% \mathrm{Cl}=0.20-0.62 ; \mathrm{p}=0.0004$ ) within the blanking period. ${ }^{11}$

Dabigatran and rivaroxaban are new oral anticoagulants that are eliminated through the kidneys. Their use in dialysis patients is discouraged in prior studies because these drugs can bioaccumulate to precipitate inadvertent bleeding. This study aimed to determine whether prescription of dabigatran or rivaroxaban was occurring in the dialysis population and whether such practices were safe. Major bleeding rate was higher in patients who were treated with dabigatran and revaroxaban, then warfarin and aspirin (respectively 83.1, 68.4, 47.1, 35.9 events/100 patients per year). The risk of haemorrhagic death was even greater with dabigatran (CA 1.78; $95 \% \mathrm{Cl}, 1.18-2.68$; $\mathrm{p}=0.006$ ) and rivaroxaban ( $\mathrm{CA} 1.71 ; 95 \% \mathrm{Cl}, 0.94-3.12 ; \mathrm{p}=0.07$ ) relative to warfarin. In covariate adjusted Poisson regression, dabigatran (CA 1.48; $95 \% \mathrm{Cl} 1.21-1.81 ; p=0.0001$ ) and rivaroxaban (CA 1.38; $95 \% \mathrm{Cl}$ 1.03-1.83; $\mathrm{p}=0.04$ ) were associated with a higher risk of hospitalisation or death from bleeding compared with warfarin."
1. Wyse DG, Waldo AL, DiMarco JP, et al., A comparison of rate control and rhythm control in patients with atrial fibrillation N Engl J Med, 2002;347:1825-33.

2. Van Gelder IC, Hagens VE, Bosker HA, et al., A comparison of rate control and rhythm control in patients with recurrent persistent atrial fibrillation, N Eng/ I Med, 2002:347:1834-40.

3. Falk RH, Atrial fibrillation, N Eng/ J Med, 2001;344:1067-78.

4. Connolly SJ, Appropriate outcome measures in trials evaluating treatment of atrial fibrillation, Am Heart J, 2000;139:752-60.

5. Turakhia MP, Santangeli P, Winkelmayer WC, et al., Increased mortality associated with digoxin in contemporary patients with atrial fibrillation: findings from the TREAT-AF study, J Am Coll Cardiol, 2014;64:660-8.

6. Washam JB, Stevens SR, Lokhnygina Y, et al., Digoxin use in patients with atrial fibrillation and adverse cardiovascular outcomes: a retrospective analysis of the Rivaroxaban Once Daily Oral Direct Factor Xa Inhibition Compared with Vitamin K Antagonism for Prevention of Stroke and with Vitamin K Antagonism for Prevention of Stroke and 2015;385:2363-70.

7. Ulimoen SR, Enger S, Pripp AH, et al., Calcium channel blockers improve exercise capacity and reduce $\mathrm{N}$-termina Pro-B-type natriuretic peptide levels compared with beta-blockers in patients with permanent atrial fibrillation Eur Heart J, 2014;35:517-24.

8. Mont L, Bisbal F, Hernández-Madrid A, et al.; SARA investigators, Catheter ablation vs. antiarrhythmic drug treatment of persistent atrial fibrillation: a multicentre, randomized, controlled trial (SARA study), Eur Heart s, 2014;35:501-7.

9. Morillo CA, Verma A, Connolly SJ, et al.: RAAFT-2 Investigators,
Radiofrequency ablation vs antiarrhythmic drugs as firstline treatment of paroxysmal atrial fibrillation (RAAFT-2): a randomized trial, JAMA, 2014;311:692-700.

10. Atienza F, Almendral J, Ormaetxe JM, et al.. RADAR-AF Investigators, Comparison of radiofrequency catheter ablation of drivers and circumferential pulmonary vein isolation in atrial fibrillation: a noninferiority randomized multicenter RADAR-AF trial, J Am Coll Cardiol, 2014;64:2455-67.

11. Darkner $S$, Chen $X$, Hansen J, et al., Recurrence of arrhythmia following short-term oral AMIOdarone after CATheter ablation for atrial fibrillation: a double-blind, randomized, placebo-controlled study (AMIO-CAT trial), Eur Heart J, 2014;35:3356-64.

12. Chan KE, Edelman ER, Wenger JB, et al., Dabigatran and Rivaroxaban use in atrial fibrillation patients on hemodialysis, Circulation, 2015;131:972-9. 